"Mircea cel Batran" Naval Academy Scientific Bulletin, Volume XIX - 2016 - Issue 1

Published by "Mircea cel Batran" Naval Academy Press, Constanta, Romania // The journal is indexed in:

PROQUEST / DOAJ / DRJI / JOURNAL INDEX / I2OR / SCIENCE LIBRARY INDEX / Google Scholar / Crossref /

Academic Keys / ROAD Open Access / OAJI / Academic Resources / Scientific Indexing Services / SCIPIO

\title{
SIMULATION BASED EMERGENCY RESPONSE TRAINING
}

\author{
Marko PERKOVIC ${ }^{1}$ \\ Marian RISTEA ${ }^{2}$ \\ Kinga LAZUGA ${ }^{3}$ \\ ${ }^{1} \mathrm{PhDc}$, University of Ljubljana, Faculty of Maritime Studies and Transport, Pot pomorscakov 4, 6320 \\ Portoroz, Slovenia, Email: marko.perkovic@fpp.uni-lj.si \\ ${ }^{2}$ Assoc. Prof. PhD "Mircea cel Batran" Naval Academy, Marine Engineering Faculty, Constanta, Romania \\ ${ }^{3} \mathrm{PhDc}$ Maritime University of Szczecin, LNG \& LCH Simulation Center
}

\begin{abstract}
Advanced simulators are rapidly becoming educational and research necessities at maritime school, a tool that has come to replace time onboard ship, bring stakeholders together on research projects, and evaluating emergency situations. This particular paper suggests some possibilities simulation offers when maritime accidents and/or incidents have already occurred. Although proper simulation training provides an accessible introduction to background theories through the realistic operations of the simulator, at the same time it can provide a means of introducing students to applications regarding current crises. This paper will demonstrate how simulators (ship handling, communication, cargo handling, terminal, vessel traffic service and engine room) can, together with the Potential Incident Simulation Control and Evaluation System (PISCES II), form valuable tools for education, training and real situation response when major pollution at sea is a threat or already exists. In this particular case the very realistic scenario of an oil spill is created.
\end{abstract}

Keywords: Marine Simulators, accidents, operational pollution, cargo handling, integration, backtracking

\section{Introduction into operational and accidental} pollution

Often research papers open as if they were promotional brochures, pointing out that an enormous percentage of world trade is carried out by sea and that in relative terms commercial shipping is relatively safe and clean. To whatever extent that may be true, intensive and costly efforts are required to maintain this status [1], which becomes more questionable as the scientific community re-evaluates the limits of human impact on the environment; on top of that, for seafarers, the business has become one of the most dangerous in the world. Perhaps it is a bit early to panic about, for instance, oil pollution, but it is certainly a good idea to intensify research efforts. The most common cause of pollution by ships comes from human error or intent - when the ship's crew does not follow the strict regulations and chooses to dump or deliberately discharge oil and chemicals into the sea. Although accidental spills from cargo and bunkers potentially constitute the worst environmental impact from shipping, regular operations generate emissions and wastes that are more or less continuously released to the marine environment [2]. Figure 1 illustrates the shipping intensity (traffic density obtained from AIS for the period of 30 days in June 2011) in part of the Mediterranean and Atlantic, indicating how the main environmental aspects arise from normal operations.

The regulatory measures adopted by the IMO where the original mandate was mainly focused

DOI: 10.21279/1454-864X-16-I1-015

(C) 2015. This work is licensed under the Creative Commons Attribution-Noncommercial-Share Alike 4.0 License. on maritime safety - have proven successful in reducing vessel-sourced pollution.

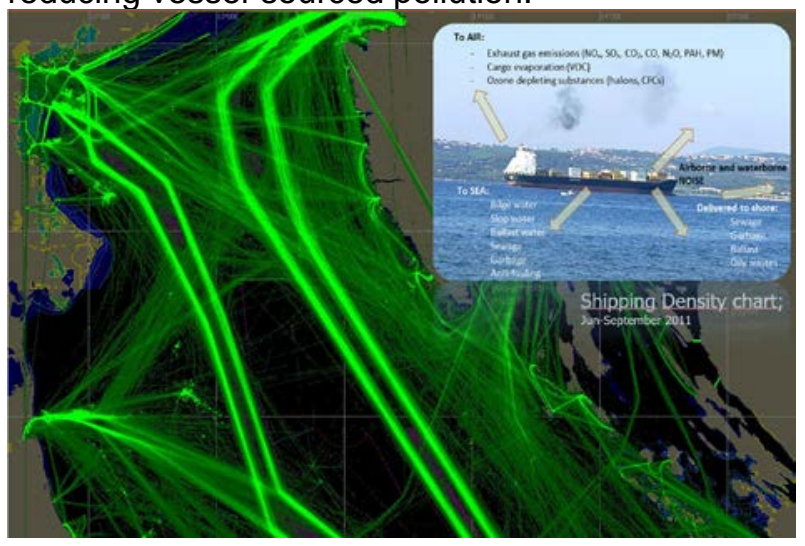

Fig.1 Main environmental aspects arising from normal operations

In 1973, the IMO adopted the International Convention for the Prevention of Pollution from Ships (MARPOL). Significant reductions of pollution have been achieved by addressing technical and operational issues. Considering operational oil pollution, the many innovations introduced by MARPOL on allowable discharges of bilge water through the oily water separator (with the well-known 15 ppm standard), or oily waters from the cargo tanks, through the oil discharge and monitoring system, have contributed greatly to a noticeable decrease in the pollution of the world's seas [1], while remaining illicit activities are still at a relatively high level. The European Maritime Safety Agency (EMSA) 
has established an operational system based on the use of near real time (NRT) space-borne imagery to support and integrate aerial surveillance in the detection of oil pollution. The platform is known as the CleanSeaNet (CSN) system, which uses Synthetic Aperture Radar (SAR) images. Having already acquired and analysed more than 15.000 images (since 2007), this service is confirming that oil is still illicitly pumped out across all European seas. Radar can only detect concentrations above 15 ppm, meaning that almost "all" positive indications in the Mediterranean are illicit. There are some special cases - like the discharging of vegetable oil - where a low concentration is allowed, if it less than $200 \mathrm{ppm} 12 \mathrm{~nm}$ from the nearest land. Global vegetable oil shipment is almost $60 \mathrm{mil}$. tonnes per year or equal to $1 / 3$ of the overall chemical tanker trade. There is a special standard related to the offshore industry where "production water" may be discharged if the maximum oil content is below $40 \mathrm{mg}$ per litre (ppm) measured as an average in any calendar month and the content dies not at any time exceed $100 \mathrm{mg}$ per litre. This is the reason we can't say that all detected indications are illicit.

The If figure 2 shows 2,630 possible oil spills detected in the presented area, where "red" coloured spots (1,565 detections) are class A spills while the rest of them belong to $B$ class detections (1,065 green coloured spots - are those with lower detection confidence). Figure 3 shows a time series overall detections and the average number of detections per square kilometre. One can conclude that various international activities along with an especially intense and rigorous vessel inspections program by the EU and probably mostly CSN contributed to environmental awareness. The average number of detections has been significantly reduced, almost cut in half considering the difference between the average of the last two years compared with the initial two years $(4.26+3.89 / 10.77+7.61=44 \%)$

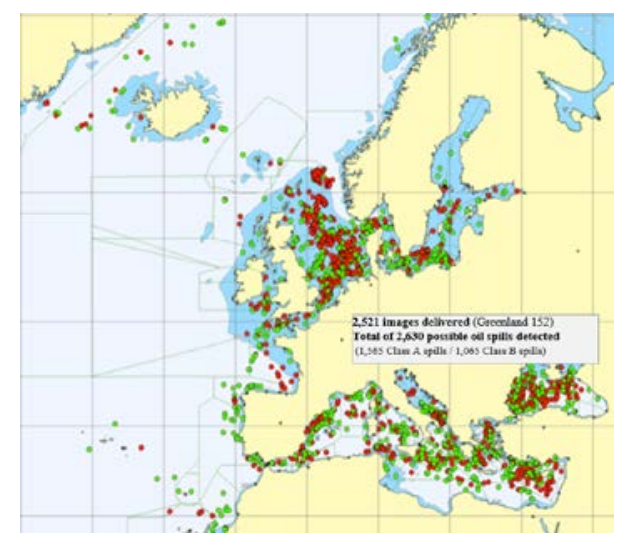

Fig.2 CleanSeaNet oil spill detections and surveillance in European waters

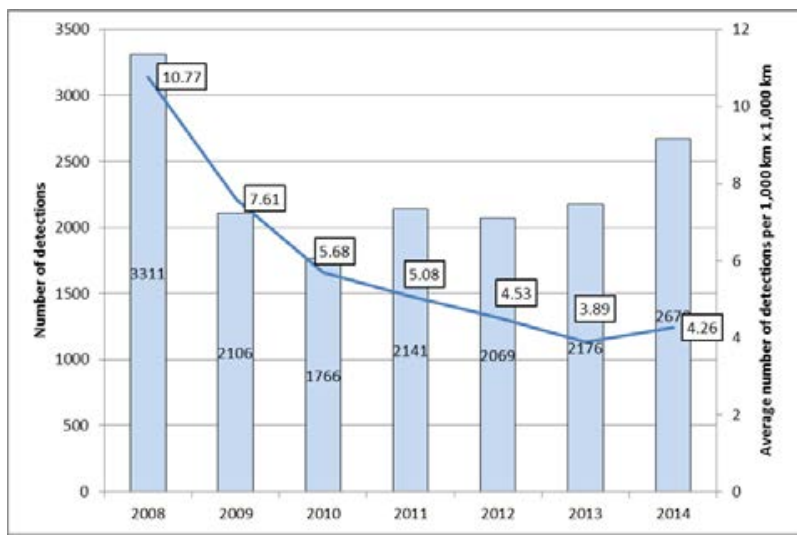

Fig.3 CleanSeaNet detections and average number of detection per milion $\mathbf{k m}^{2}$

\section{Operational and accidental pollution}

\subsection{Operational pollution}

Marine oil pollution by vessels, termed "operational oil pollution", includes various types of discharges of oil and oily mixtures, as a result of ships' daily routine operations. Some of these, such as oily ballast water and tank washing residues, relate only to tankers. All types of ships, however, may cause pollution through discharging oil into the sea coming from engine room wastes, bilge waters, and, in rare cases, used oil [3]. Before being burnt, fuel oil must be centrifuged, a process by which residues are stored in a sludge tank. The sludge should be delivered to port facilities. However, in practice, ships do not always unload residues in ports, rather the unrecorded discharges are accomplished through the use of a "magic pipe" [4] that connected the ship's purifier sludge tank with the ship's bilge holding tank, the contents of which are then pumped overboard without first being processed through required pollution prevention control equipment (ODM) designed to detect and prevent discharges containing more than 15 parts per million oil. Figure 4 demonstrates such real illegal or even criminal act (violation) on board the ship using magic pipe/bypass in the engine room while simulating such an event using either a liquid cargo handling simulator or engine room simulator is shown in figure 5 .

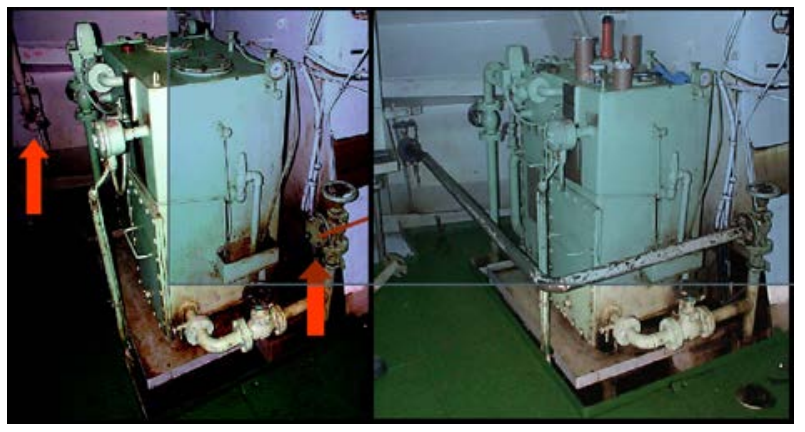

Fig.4 Direct discharge; bypassing the oilywater separator system 


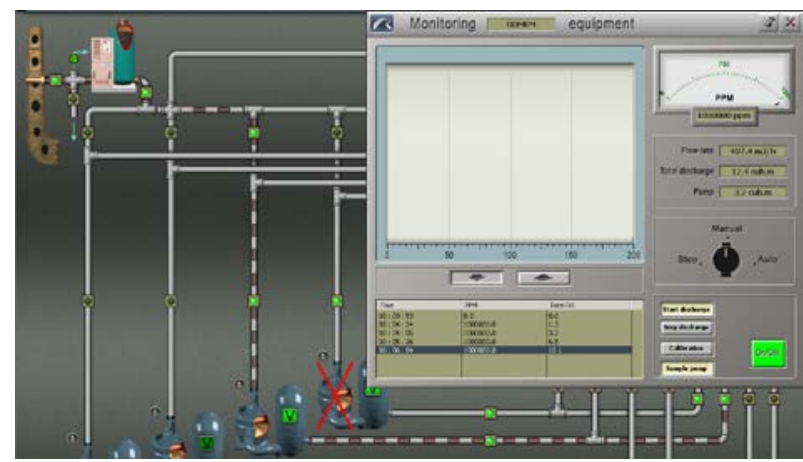

Fig.5 Simulation based violation of the act to prevent pollution from ships

The next figure (Fig. 6) shows a case of interest CleanSeaNet detection on 22 March in Croatian waters. The spill was detected approximately 5 hours after the discharge. A possible source (MMSI number) was reported by the CSN service provider [5]. The vessel track was available in an alert report based on AIS information integrated into the CSN system. Researchers performed hindcast modelling using an advanced oil spill simulator (PISCES 2) [6] and successfully backtracked the potential polluter, an identification which was further vetted by the Slovenian port state inspector. Finally, an overriding factor message in Thetis regarding a possible instance of pollution in Croatian waters was entered. The inspector found evidence of a discharge of oily products:

- an OWS line containing oil residues

- Oil spots on the starboard side hull (about 10 square meters)

The master and the company were fined 4,600 Euros. The ship was not detained.

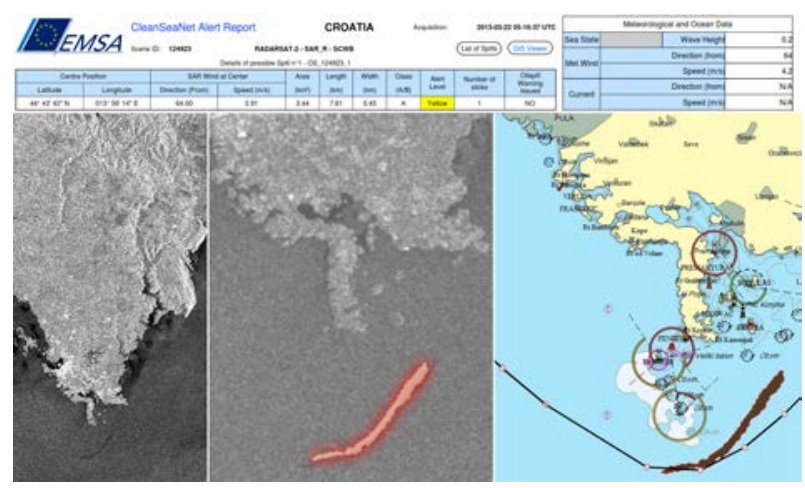

Fig.6 Illicit pollution case

\subsection{Accidental pollutions}

Following the latest report issued by ITOF [7] the vast majority of spills are small and medium size (together constituting 95\%). Large spills account for the remaining $5 \%$ of all the incidents recorded and the occurrence of these incidents has significantly decreased over the last decades.

DOI: 10.21279/1454-864X-16-I1-015

(c) 2015. This work is licensed under the Creative Commons Attribution-Noncommercial-Share Alike 4.0 License.
While the primary source of large spills are groundings (33\%), collisions $(30 \%)$, hull failures $(13 \%)$, fire and explosion (11\%) equipment failures (4\%), and other/unknown causes includes events such as heavy weather damage and human error. Small and medium spills generally occurred during loading and discharging (29\%), which normally take place in ports and oil terminals. The cause of the majority of these spills is unknown. Due to the in shore the vicinity, even small or eventually medium size spills pose a high risk [8]. To demonstrate, we can describe a minor operational or accidental spill that occurred during 2006 in the port of Koper, in which less than 5 tons of sludge was discharged during the night in the first basin, from where it rapidly spread to the city centre beach. Cleaning costs came to more than one million US dollars. To mitigate such risks a modern simulation based approach may be applied to access the risk, to develop appropriate contingency planning, and to identify adequate equipment and structures. Such a system may also be used for education and training [9].

Figure 7 shows an integrated terminal and liquid cargo handling simulator where an oil spill accident occurs at manifolds, spill is dynamically changed and affected by a change of weather conditions.

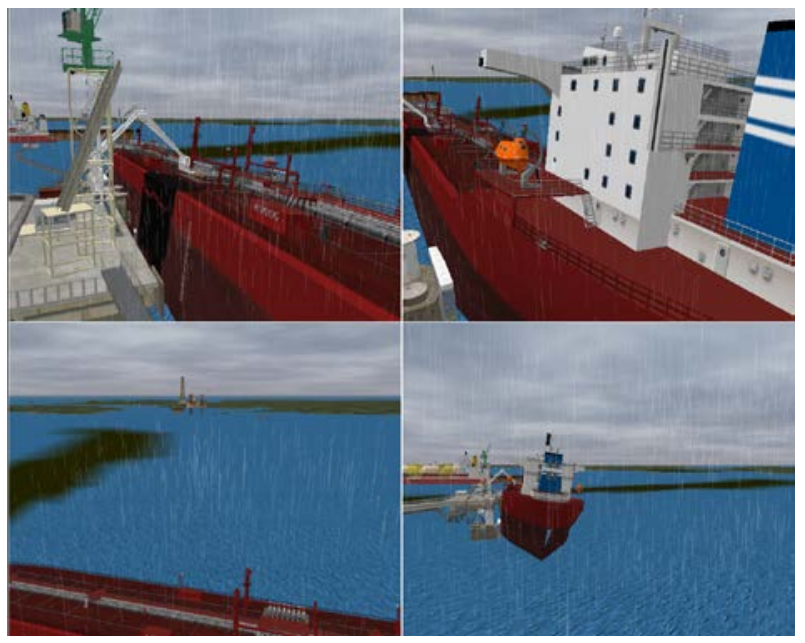

Fig.7 Integrated simulation approach; accidental pollution at tanker terminal

\section{Simulation based emergency response training}

As noted previously, environmental protection is extensively regulated and monitored/controlled, but intentional pollution yet occurs. The IMO has taken a step further by stressing marine environmental awareness and the coordination of activities during emergencies (safety, security, pollution prevention) in a revised STCW convention (the Manila Amendments). The IMO also presented its vision of a framework for Sustainable Maritime Development, and its plans 
to develop Sustainable Development Goals for the maritime industry, focusing on eight pillars: 1. safety culture and environment stewardship, 2. energy efficiency, 3. new technology and innovation, 4. maritime education and training, 5. maritime security and anti-piracy actions, 6 . maritime traffic management, 7. maritime infrastructure development, 8. adoption and implementation of global standards by the IMO.

Integrated simulators are an appropriate tool for a first demonstration illustrating environmental challenges, enhancing shipboard oil spill training, analysing shipboard marine pollution emergency plans, port terminal response plans and national contingency plans. To perform high-quality training, especially for imparting management skills, an integrated simulator centre that works with values computed physically and mathematically in real time is necessary. Different scenarios involving various malfunctions can be illustrated through such a system, even, and importantly, those unlikely to occur - and when students observe the use of simulation applied to real events they are clearly, in our experience, better able to understand the importance of this latter stipulation.

\subsection{Integrated simulator and Cases}

Simulator centres may be configured on different levels. The complex integrated simulator centre at the Faculty of Maritime Studies [10] is linked through the secure VPN to external traffic sources such as the Automatic Identification System based VTS system at the Slovenian Maritime Administration office, the RADAR based VTS system at the Slovene littoral marine police station and the oceanographic and meteorological source at the marine biology station. This configuration enables effective training and competency assessment of deck/engine officers, loading masters and other stakeholders. The centre is also invaluable for research and investigation into port development, ship manoeuvring and improving ship and port safety and efficiency [11]. Figure 8 shows the integration of pollution described in figure 7 and further modelled with an oil spill simulator and for distribution among stakeholder exported as a Google Earth GIS inf.

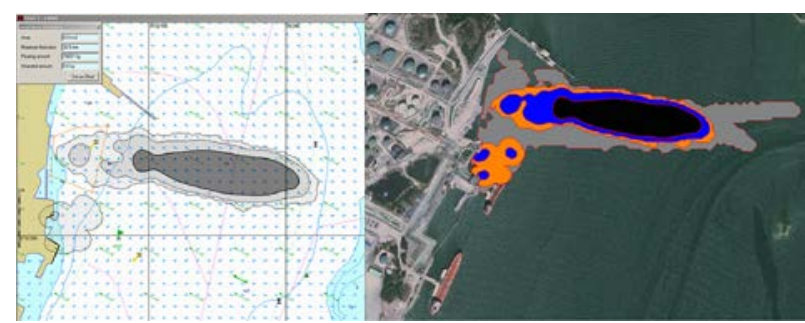

Fig.8 Integration of a liquid cargo handling simulator with a port terminal operation and oil spill simulator
There are three training domains: the simulator, real time/real emergency, and a mixture of the two. Here the focus is on the realistic scenario of a passenger vessel entering a basin at too high a speed at the onset of a tramontana (this is a violent summer storm, usually of high, potentially damaging winds, the onset of which cannot be predicted within more than a few hours) and thus colliding with a moored general cargo vessel. At least twice, that we are aware of, pilots saved their vessels in just such a situation by abandoning tugs and increasing speed in order to find shelter from the wind as quickly as possible. Given the generally excellent manoeuvrability of passenger vessels with their bow and stern thrusters, as well as their susceptibility to wind because of their height, it is not unlikely that a pilot might try exactly such an act in such a situation. A number of factors, including too great a roll, the squat effect, and the general difficulty of controlling the speed of a large vessel over extremely short periods of time in tight spaces, could lead to the collision we designed.

The results of the collision are the spilling of oil, a fire at the stern of the general cargo vessel (Figure 9) (which will not be part of our emergency simulation), and an unknown number of injuries and/or deaths. Alarms, of course, will be set off, but we assume that the master of either vessel survives in good order, immediately recognizes the severity of the situation, and makes immediate contact with the MRCC, informing them of the accident. The ship's crew meanwhile monitors the oil spill, providing relevant information. The MRCC commands the immediate stop of the ship, which has already occurred, but is worth mentioning for their thought is that the oil spill must be limited to the smallest area possible, even within a virtually enclosed basin. A spillage of oil in the quantity of more than $10 \mathrm{~m} 3$ is foreseen, so the MRCC informs the Regional Notification Centre (RNC), which acts as coordinator and dispatches information to all relevant competent bodies. Due to the significant amount of spillage, the RNC notifies the regional Head Quarters (HQ) of the Civil Protection and Disaster Relief (ACPDR). The regional $\mathrm{HQ}$ acts as coordinator, within the ACPDR national HQ, by telephone. Regional HQ calls up the other services that have resources for the gradual limiting of oil spills: the coastal safeguard service - SVOM - under the Environmental Agency (EA), the marine police, the navy and the tugboat company. Each of the commanders arrives at the appropriate moment to his work station. Marine biology experts may assist in case beaching of the oil is foreseen or dispersants must be used. The local firefighting brigade is also activated for controlling the fire and preventing explosions on-board the ships or in case a special cleaning plan is activated if the oil spill has to be burned. The on-scene commander 
embarks with the crew on virtual bridge 1 , representing the SAR ship under command of the Maritime Administration Directorate.
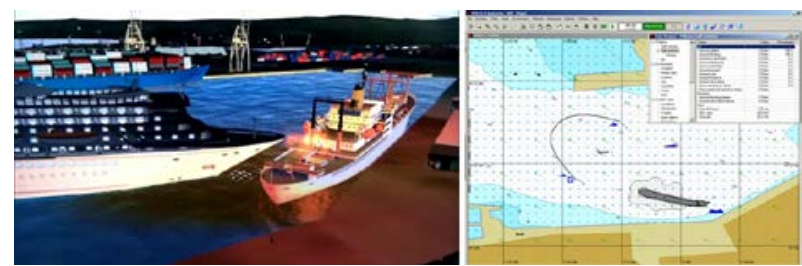

Fig.9 Integration of navigational (ship handling including VTS), GMDSS, engine room and oil spill simulator

The EA and SVOM officials embark on the tugboats that tow the barge with the oil spill combating equipment. Civil protection calls the simulation centre, where the researchers, marine biologists, and representatives from the EA have prepared a model of the oil spill drift based on the forecast of winds and currents. The PISCES simulator connects to the VTS (NaviHarbour application) receiver and so locates the ships in distress. The current state of the wind, currents, salinity and temperature are loaded into the GIS system automatically. The quantity and type of the spilled oil is provided by the ship and the initial simulating area is given by the SAR vessel that constantly transmits its position with an AIS device while navigating around the border of the spill. The rescue commander estimates that the required time for the deployment of booms will be one hour and a simulation of the expected spill position after this time is made. Regarding the configuration, the strike team agrees on the type of equipment and its position. The simulated situation is seen on a monitor at each of the operating stations and on the visualization system of the ship handling simulator. After two hours a satellite picture of the oil spill situation is received and included into the PISCES program, correcting the oil spill position for the necessary time of the simulation. The whole procedure of rescue and communication is archived with video cameras and the entire system is thoroughly interactive.

\section{CONCLUSION}

The maritime industry, a potential source of catastrophic environmental damage, is in theory quite well regulated. Unfortunately, regulations do not enforce themselves and intentional/operational pollution remains problematic, as does the refusal of some ship owners to take proper care to prevent such spills as are caused by hull deficiencies, for instance. The mathematics behind the problem are quite simple: as long as polluting or failing to prevent pollution is vastly cheaper than doing everything possible to maintain a clean environment, those drawing income from control of maritime commerce will tend to behave in ways increasingly unacceptable for the environment and those who desire to maintain it at a high standard. We have described an identified polluter that was fined $4600 €$ and an instance of pollution that cost over one million dollars. Until the economics of prevention become a factor in European enforcement, we can expect pollution to remain at a high level, and that level to become farther and farther from that which is necessary to preserve a healthy global environment.

The IMO is acutely aware of these problems and is avidly attempting to address the issues. Our role is in the instruction of seafarers, future researchers, port authorities, and any and all other stakeholders through the use of integrated simulation. The use of simulation has of course been established as a necessary research and instructional tool. From this paper one can see that while one particular scenario is described in detail, the possibilities are virtually limitless. And in this same section it is readily apparent that there are a variety of potential uses that could all be described extensively for purposes of disseminating research and/or for pedagogical uses. The case described in section three is just one of many scenarios the simulation centre can effectively examine in order to add to the prospective safety of vessels and of the environment, being used for port design, weather emergency scenarios, risk assessment, collision avoidance, SAR, contingency planning, and so on.

We may try to look forward to a time when simulation is no longer necessary for the pursuit of environmental polluters, but our vision does not extend beyond the horizon obscuring such hopes; What is more, much work will be required to keep up with the expanding industry, particularly in regard to safety and the protection of the environment.

\section{BIBLIOGRAPHY}

[1] IMO and Environment, International Maritime Organization, (2011). www.imo.org/OurWork/Environment/Documents/IMO\%20and\%20the\%20Environment\%202011.pdf

[2] Shipping, world trade and the reduction of $\mathrm{CO}_{2}$ emmisions, International Chamber of Shipping, http://www.shippingandco2.org/CO2-Flyer.pdf

[3] Ferraro G., Bernardini A., David M., Meyer-Roux S., Muellenhoff O., Perkovic M., Tarchi D., Topouzelis K., "Towards an operational use of space imagery for oil pollution monitoring in the Mediterranean basin: A demonstration in the Adriatic Sea", Marine Pollution Bulletin, Volume 54, Issue 4, pp 403-422, (2007) 
[4] Pasfield B., Rindfleisch E., "Finding the magic pipe: do seamen have constitutional rights when a U.S. Coast Guard boarding turns criminal?", U.S.F. Maritime Law Journal, Vol. 22 No.1, pp. 23-38, (2010).

[5] EMSA, CSN Schedule of new releases, $12^{\text {th }}$ CleanSeaNet User Group, Lisbon, 10-11 April 2013

[6] Perkovic M., Greidanus H., Muellenhoff O., Ferraro G., Pavlakis P., Cosoli S. and Harsch R., "Marine polluter identification: Backtracking with the aid of satellite imaging", Fresenius Environmental Bulletin, Vol. 19, No 10b, pp 2426-2432, (2010).

[7] ITOPF, Oil tanker Spill Statistics 2012, The international tanker owners pollution federation limited, http://www.itopf.com/information-services/publications/documents/StatsPack 000.pdf

[8] Lazuga K., Gucma, L.,, Perkovic, M., Optimal allocation of response resources model verification on the example of oil spill on the Pomeranian Bay. V: Soares, C. Guedes (ur.), Dejhalla, Roko (ur.), Pavletić, Duško (ur.). Towards green marine technology and transport : proceedings. Leiden: Taylor \& Francis Group, 2015, pp 279-283.

[9] Gucma L., Juszkiewicz W., Łazuga K., "The Method of Optimal Allocation of Oil Spill Response in the Region of Baltic Sea", International Journal on Marine Navigation and Safety of Sea Transportation, Vol. 6, No. 4, pp. 489-493, (2012).

[10] Perkovic M., Suban V., Petelin S., David M., "Complex maritime system simulator centre for advance training and education". 4th international science symposium, 19-21. April 2006, Portoroz, Slovenia, pp. 835-839, (2006).

[11] Perkovic M., Harsch R., Suban V., Vidmar P., Nemec D., Muellenhoff O., Delgad L. "The Use of Integrated Maritime Simulation for Education in Real Time", International Maritime Lecturers' Association, 16th Conference on MET, IMLA 2008, 14-18 October, Izmir, Turkey, pp.461-478, (2008) 